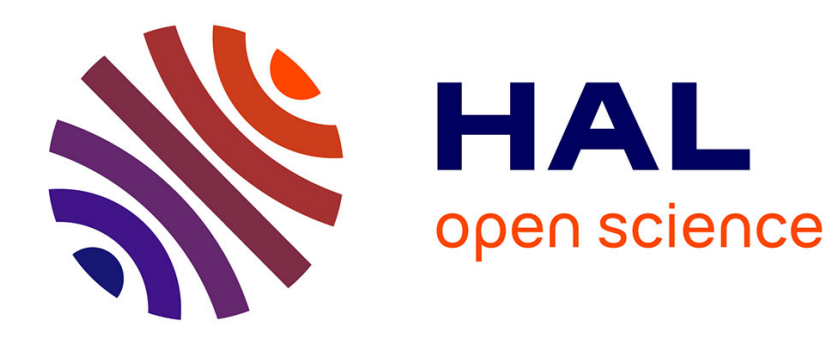

\title{
Restricted-trace approximation for nuclear antiferromagnetism
}

\author{
M. Goldman, G. Sarma
}

\section{To cite this version:}

M. Goldman, G. Sarma. Restricted-trace approximation for nuclear antiferromagnetism. Journal de Physique, 1975, 36 (12), pp.1353-1362. 10.1051/jphys:0197500360120135300 . jpa-00208383

\section{HAL Id: jpa-00208383 https://hal.science/jpa-00208383}

Submitted on 1 Jan 1975

HAL is a multi-disciplinary open access archive for the deposit and dissemination of scientific research documents, whether they are published or not. The documents may come from teaching and research institutions in France or abroad, or from public or private research centers.
L'archive ouverte pluridisciplinaire HAL, est destinée au dépôt et à la diffusion de documents scientifiques de niveau recherche, publiés ou non, émanant des établissements d'enseignement et de recherche français ou étrangers, des laboratoires publics ou privés. 


\author{
Classification \\ Physics Abstracts \\ $1.660-5.450-8.510$
}

\title{
RESTRICTED-TRACE APPROXIMATION FOR NUCLEAR ANTIFERROMAGNETISM
}

\author{
M. GOLDMAN and G. SARMA \\ Service de Physique du Solide et de Résonance Magnétique, \\ Centre d'Etudes Nucléaires de Saclay, BP № 2, 91190 Gif-sur-Yvette, France
}

(Reçu le 10 juillet 1975, accepté le 26 août 1975)

\begin{abstract}
Résumé. - Nous utilisons dans ce travail l'approximation de la trace restreinte pour prédire quelques propriétés des structures antiferromagnétiques nucléaires : aimantation des sous-réseaux, susceptibilités longitudinale et transversale. Cette étude est spécialisée à une structure antiferromagnétique particulière observée dans le fluorure de calcium. Bien que non parfaites, les prédictions de cette théorie sont en accord qualificatif avec les résultats expérimentaux et constituent un important progrès sur l'approximation du champ de Weiss combinée à celle des hautes températures.

L'approximation du second ordre n'apporte pas de progrès notable sur celle du premier ordre.

Abstract. - We use in this work the restricted-trace approximation to predict several properties of nuclear antiferromagnetic structures : sublattice magnetization, longitudinal and transverse susceptibilities. This study is specialized to a particular antiferromagnetic structure, observed in calcium fluoride. Although not perfect, the predictions of this theory are in qualitative agreement with the experimental results, and constitute an important improvement on the Weiss-field approximation combined with the high-temperature approximation.

The second order approximation is not significantly better than the first-order one.
\end{abstract}

1. Introduction. - Experimental evidence for the existence of nuclear dipolar magnetic ordering, either antiferromagnetic or ferromagnetic, has been reported in a number of articles [1-5]. In reference [5] were described in detail the principle of production of nuclear magnetic ordering in the rotating frame and the prediction of the ordered structures through the local Weiss-field approximation. For simple cubic systems of nuclear spins $\frac{1}{2}$, such as the ${ }^{19} \mathrm{~F}$ spin system in calcium fluoride, this method led to the prediction of three different antiferromagnetic structures. The prediction of the properties of the antiferromagnetic structures as a function of field and entropy, was made through a makeshift theory consisting of the Weissfield approximation supplemented, in the paramagnetic phase, by a high temperature expansion for spintemperature theory. This procedure, although lacking in thermodynamic consistency, yields results which are in reasonable semi-quantitative agreement with the experimental observations. Its main disadvantage is that the paramagnetic and the antiferromagnetic phases are treated independently, and that local spinspin order is taken partially into account only in the former phase.

The purpose of this article is to describe the application of the restricted-trace method $[6,7]$ to nuclear dipolar antiferromagnetism. This method is closely related to the former theory. Its advantages are that it is consistent from the point of view of thermodynamics, that it takes into account local spin-spin order even in the ordered phase and that it covers with a single formalism both domains of paramagnetism and of magnetic ordering.

Firstly, let us briefly recall the principle of the production of nuclear magnetic ordering in the rotating frame. This ordering is achieved in a twostep process. In the first step the nuclear spin system is dynamically polarized in high field by the Solid Effect, which results in an important decrease of its entropy. One then performs an adiabatic demagnetization of the nuclear spins in the rotating frame, which has the effect of removing the effective field experienced by the nuclei, while retaining the low value of their entropy. In zero effective field, that is, at the centre of the fast passage, the Hamiltonian controlling the evolution of the spin system consists of the familiar truncated, or secular, dipole-dipole Hamiltonian $\mathfrak{H}_{\mathrm{D}}^{\prime}$. Provided that the entropy is sufficiently low, the nuclear spin system can undergo, in low effective field, a transition to a magnetically ordered state. The structure of this ordered state depends on the form of the Hamiltonian $\mathcal{H}_{\mathrm{D}}^{\prime}$, which in turn depends on the orientation of the external magnetic field with respect to the crystalline axes. It also depends on the sign of 
the spin temperature, which can be made positive or negative at will by starting the adiabatic fast passage with the effective field either parallel or antiparallel to the nuclear magnetization, respectively.

- The experimental investigation of nuclear magnetic ordering has been to a large extent devoted to the antiferromagnetic phase of the ${ }^{19} \mathrm{~F}$ spins of $\mathrm{CaF}_{2}$, produced by adiabatic demagnetization in the rotating frame at negative spin temperature with the external magnetic field parallel to the [100] axis. The structure predicted in that case is depicted on figure 1 . It is to this structure that the restricted-trace method will be applied in the following section. Its predictions will be compared with earlier theoretical predictions, as well as with existing experimental data.

2. Principle of the restricted-trace method. - The method of the restricted trace was invented by Kirkwood [6,7], who used it for the study of ordering in binary alloys. We recall its principle, as applied to an antiferromagnet.

We consider a system of spin-spin Hamiltonian $\mathcal{J}_{\mathbf{D}}^{\prime}$ which undergoes at low temperature a transition to an antiferromagnetic state of known structure : it consists of two sublattices, labelled $\mathcal{A}$ and $\mathcal{B}$ respectively, which in zero field carry opposite magnetizations along a direction $\mathrm{O} z$. We call $I_{i}$ and $S_{\mu}$ the spins belonging to sublattices $\mathcal{A}$ and $\mathcal{B}$ respectively; and $N$ the number of spins in each sublattice. In the presence of an external field parallel to $\mathrm{O} z$, the polarizations of the sublattices are $p_{\mathrm{A}}$ and $p_{\mathrm{B}}$, respectively. We restrict ourselves to spins $\frac{1}{2}$, so that :

$$
p_{\mathrm{A}}=2\left\langle I_{z}^{i}\right\rangle \text { and } p_{\mathrm{B}}=2\left\langle S_{z}^{\mu}\right\rangle \text {. }
$$

These polarizations may be equal, if the conditions of field and temperature are such that the system is paramagnetic. They become different when the system is ordered.

If $\beta$ is the inverse temperature of the system, its partition function is :

$$
Z=\operatorname{Tr}\{\exp (-\beta \mathcal{H})\}
$$

where the Hamiltonian consists of a Zeeman term plus a spin-spin term :

$$
\mathscr{H}=\Delta\left(I_{z}+S_{z}\right)+H_{\mathrm{D}}^{\prime} .
$$

We split the Hilbert space into a sum of subspaces, each of which is spanned by eigenkets of both $I_{z}=\sum_{i} I_{z}^{i}$ and $S_{z}=\sum_{\mu} S_{z}^{\mu}$, belonging to well-defined eigenvalues $\left\langle I_{z}\right\rangle=\frac{1}{2} N p_{\mathrm{A}}$ and $\left\langle S_{z}\right\rangle=\frac{1}{2} N p_{\mathrm{B}}$, different for each subspace, and ranging from $-\frac{1}{2} N$ to $\frac{1}{2} N$. The partition function can then be written as a sum of traces restricted to the various subspaces :

$$
Z=\sum_{\left(p_{\mathbf{A}}, p_{\mathbf{B}}\right)} \operatorname{Tr}^{\prime}\{\exp (-\beta \mathcal{H})\}
$$

The hypothesis made by Kirkwood is that the restricted traces exhibit a sharp maximum at particular values of $p_{\mathrm{A}}$ and $p_{\mathrm{B}}$, which are those existing in the ordered system. We call in the following

$$
\operatorname{Tr}^{\prime}\{\exp (-\beta \mathcal{H})\}
$$

the restricted trace which is maximum. The sum (4) is made of $2 N+2$ different restricted traces, and we have :

$$
\begin{aligned}
\operatorname{Tr}^{\prime}\{\exp (-\beta \mathcal{H})\}<Z<(2 N & +2) \\
& \times \operatorname{Tr}^{\prime}\{\exp (-\beta \mathcal{H})\}
\end{aligned}
$$

whence :

$$
\ln Z=\ln \operatorname{Tr}^{\prime}\{\exp (-\beta \mathcal{H})\}+O(\ln N)+O(1) .
$$

Furthermore, since $\ln Z$ is proportional to the number of particles we have in the thermodynamic limit, that is when $N \rightarrow \infty$ :

$$
\ln Z \simeq \ln \operatorname{Tr}^{\prime}\{\exp (-\beta J)\}
$$

In fact there will be in general two couples $\left(p_{\mathrm{A}}, p_{\mathrm{B}}\right)$ with equal sharp values of the restricted trace. One will correspond to $p_{\mathrm{A}}=p^{\prime}$ and $p_{\mathrm{B}}=p^{\prime \prime}$, and the other to $p_{\mathrm{A}}=p^{\prime \prime}$ and $p_{\mathrm{B}}=p^{\prime}$. We choose arbitrarily one of them, thus imposing the symmetry breaking which is known to occur in an antiferromagnetic transition. The corresponding error for $\ln Z$ is equal to $\ln 2$, which is negligible.

The procedure is then to compute the restricted trace as a function of $p_{\mathrm{A}}$ and $p_{\mathrm{B}}$, and to determine the latter by the condition that they maximize this restricted trace. This is done as follows. We write :

$$
\operatorname{Tr}^{\prime}\{\exp (\ldots)\}=\operatorname{Tr}^{\prime} 1 \times\left[\operatorname{Tr}^{\prime}\{\exp (\ldots)\} / \operatorname{Tr}^{\prime} 1\right]
$$

where 1 is the unit operator, and we obtain :

$$
\ln Z=\ln \operatorname{Tr}^{\prime} 1+\ln \left[\operatorname{Tr}^{\prime}\{\exp (\ldots)\} / \operatorname{Tr}^{\prime} 1\right]
$$

$\operatorname{Tr}^{\prime} 1$ is equal to the number of states with the prescribed values of $p_{\mathrm{A}}$ and $p_{\mathrm{B}}$. Its logarithm is, within the factor $k_{\mathrm{B}}$, equal to the entropy in the Weiss-field approximation, $S_{\mathrm{w}}\left(p_{\mathrm{A}}, p_{\mathrm{B}}\right)$ :

$$
\begin{aligned}
\ln \operatorname{Tr}^{\prime} 1= & S_{\mathrm{w}}\left(p_{\mathrm{A}}, p_{\mathrm{B}}\right) \\
=N & \left\{2 \ln 2-\frac{1}{2}\left[\left(1+p_{\mathrm{A}}\right) \ln \left(1+p_{\mathrm{A}}\right)+\right.\right. \\
& \left.+\left(1-p_{\mathrm{A}}\right) \times \ln \left(1-p_{\mathrm{A}}\right)\right]-\frac{1}{2}\left[\left(1+p_{\mathrm{B}}\right)\right. \\
& \left.\left.\times \ln \left(1+p_{\mathrm{B}}\right)+\left(1-p_{\mathrm{B}}\right) \ln \left(1-p_{\mathrm{B}}\right)\right]\right\} .
\end{aligned}
$$

In the case we are dealing with, the spin-spin Hamiltonian, which is a truncated dipole-dipole Hamiltonian, commutes with $\left(I_{z}+S_{z}\right)$. Furthermore, since the subspace in which we calculate the restricted 
trace consists of degenerate eigenkets of both $I_{z}$ and $S_{z} \quad$ that is, according to eq. (10) and (9) : we have :

$$
\begin{aligned}
& \operatorname{Tr}^{\prime}\{\exp (-\beta \mathcal{H})\}= \operatorname{Tr}^{\prime}\left\{\exp \left[-\beta \Delta\left(I_{z}+S_{z}\right)\right] \times\right. \\
&\left.\times \exp \left(-\beta \mathcal{H}_{\mathrm{D}}^{\prime}\right)\right\} \\
&=\exp \left[-\frac{1}{2} \beta N \Delta\left(p_{\mathrm{A}}+p_{\mathrm{B}}\right)\right] \times \\
& \times \operatorname{Tr}^{\prime}\left\{\exp \left(-\beta \mathcal{H}_{\mathrm{D}}^{\prime}\right)\right\} .
\end{aligned}
$$

Equation (6) becomes :

$$
\begin{aligned}
\ln Z=S_{\mathrm{w}}\left(p_{\mathrm{A}}, p_{\mathrm{B}}\right) & -\frac{1}{2} \beta N \Delta\left(p_{\mathrm{A}}+p_{\mathrm{B}}\right)+ \\
& +\ln \left[\frac{\operatorname{Tr}^{\prime}\left\{\exp \left(-\beta \mathcal{H}_{\mathrm{D}}^{\prime}\right)\right\}}{\operatorname{Tr}^{\prime} 1}\right] .
\end{aligned}
$$

The values of $p_{\mathrm{A}}$ and $p_{\mathrm{B}}$ are obtained by satisfying :

$$
\frac{\partial}{\partial p_{\mathrm{A}}} \ln Z=\frac{\partial}{\partial p_{\mathrm{B}}} \ln Z=0 \text {. }
$$

For spins $\frac{1}{2}$, we have :

$$
\begin{aligned}
\frac{\partial}{\partial p_{\mathrm{A}}} S_{\mathrm{w}} & =-N \tanh ^{-1}\left(p_{\mathrm{A}}\right), \\
\frac{\partial}{\partial p_{\mathrm{B}}} S_{\mathrm{w}} & =-N \tanh ^{-1}\left(p_{\mathrm{B}}\right) .
\end{aligned}
$$

Eq. (10) then yield the following pair of coupled equations :

$$
\left.\begin{array}{rl}
\tanh ^{-1}\left(p_{\mathrm{A}}\right)=-\frac{1}{2} \beta \Delta+\frac{1}{N} \\
\times \frac{\partial}{\partial p_{\mathrm{A}}} \ln \left[\operatorname{Tr}^{\prime}\left\{\exp \left(-\beta \mathcal{H}_{\mathrm{D}}^{\prime}\right) / \operatorname{Tr}^{\prime} 1\right\}\right] \\
\tanh ^{-1}\left(p_{\mathrm{B}}\right)=-\frac{1}{2} \beta \Delta+\frac{1}{N} \frac{\partial}{\partial p_{\mathrm{B}}} \ln [\ldots]
\end{array}\right\}
$$

$$
\begin{aligned}
E=-\frac{\partial \ln Z}{\partial \beta}=\frac{1}{2} & N \Delta\left(p_{\mathrm{A}}+p_{\mathrm{B}}\right)+ \\
& +\frac{\operatorname{Tr}^{\prime}\left\{\mathscr{H}_{\mathrm{D}}^{\prime} \exp \left(-\beta \mathcal{H}_{\mathrm{D}}^{\prime}\right)\right\}}{\operatorname{Tr}^{\prime}\left\{\exp \left(-\beta \mathcal{H}_{\mathrm{D}}^{\prime}\right)\right\}} .
\end{aligned}
$$

The first term on the right-hand side is the Zeeman energy $Z$, and the second term is the dipole-dipole energy $E_{\mathrm{D}}$.

We write the entropy in the form :

$$
S=S_{\mathrm{w}}\left(p_{\mathrm{A}}, p_{\mathrm{B}}\right)+s\left(\beta, p_{\mathrm{A}}, p_{\mathrm{B}}\right)
$$

where the first term does not depend explicitly on $\beta$. Eq. (14) yields, according to eq. (16) and (15) :

$$
\frac{\partial s}{\partial \beta}=\beta \frac{\partial E_{\mathbf{D}}}{\partial \beta}
$$

whereas according to eq. (9) we find that for $\beta=0$,

$$
s\left(0, p_{\mathrm{A}}, p_{\mathrm{B}}\right)=0 .
$$

The entropy $S$ is entirely determined by eq. (11) and (15) to (18).

The approximation made by Kirkwood consists in the use of a limited expansion in $\beta$ for the logarithm on the right-hand side of eq. (9). When this expansion is limited to the term linear in $\beta$, the maximization of $\ln Z$ yields the same result for $p_{\mathrm{A}}$ and $p_{\mathrm{B}}$ as the Weissfield approximation. The whole theoretical procedure is then in fact very close to that of Bragg and Williams $[8,9]$. Terms of higher order in $\beta$ yield corrections to the Weiss-field result, arising from short-range correlations between spins.

In order that this treatment of ordering be consistent, it is necessary that in zero field (i.e. $\Delta=0$ ) there exist a critical inverse temperature $\beta_{\mathrm{c}}$ such that :

i) For $|\beta|<\left|\beta_{\mathrm{c}}\right|$ the only solution of eq. (11) is :

$$
p_{\mathrm{A}}=p_{\mathrm{B}}=0 \text {. }
$$

ii) For $|\beta|>\left|\beta_{\mathrm{c}}\right|$ the solution which maximizes $\ln Z$ corresponds to :

$$
p_{\mathrm{A}}=-p_{\mathrm{B}} \neq 0 \text {. }
$$

where $F$ is the free energy, $S$ the entropy and $E$ the energy,

$$
E=-\frac{\mathrm{d}}{\mathrm{d} \beta} \ln Z
$$

and, as a consequence of eq. (12) and (13) :

$$
\frac{\mathrm{d} S}{\mathrm{~d} \beta}=\beta \frac{\mathrm{d} E}{\mathrm{~d} \beta} .
$$

$\ln Z$ depends on $\beta$ both explicitly and implicitly, through $p_{\mathrm{A}}$ and $p_{\mathrm{B}}$. We can write :

$$
E=-\frac{\partial \ln Z}{\partial p_{\mathrm{A}}} \frac{\mathrm{d} p_{\mathrm{A}}}{\mathrm{d} \beta}-\frac{\partial \ln Z}{\partial p_{\mathrm{B}}} \frac{\mathrm{d} p_{\mathrm{B}}}{\mathrm{d} \beta}-\frac{\partial \ln Z}{\partial \beta}
$$

We recall that the sign of $\beta$ is determined by the problem under investigation. The particular case analyzed below corresponds to $\beta<0$.

3. Practical methods of calculation. - The power expansion with respect to $\beta$ of the logarithm on the right-hand side of eq. (9) is trivial :

$$
\begin{aligned}
& \ln \left[\frac{\operatorname{Tr}^{\prime}\left\{\exp \left(-\beta \mathcal{H}_{\mathrm{D}}^{\prime}\right)\right\}}{\operatorname{Tr}^{\prime} 1}\right]=-\beta \frac{\operatorname{Tr}^{\prime} \mathcal{H}_{\mathrm{D}}^{\prime}}{\operatorname{Tr}^{\prime} 1}+ \\
& +\frac{1}{2} \beta^{2}\left\{\frac{\operatorname{Tr}^{\prime}\left(\mathcal{H}_{\mathrm{D}}^{\prime 2}\right)}{\operatorname{Tr}^{\prime} 1}-\left(\frac{\operatorname{Tr}^{\prime} \mathcal{H}_{\mathrm{D}}^{\prime}}{\operatorname{Tr}^{\prime} 1}\right)^{2}\right\}+\cdots .
\end{aligned}
$$


According to a general thermodynamic result this expression, which is a part of $-\beta F$, must be proportional to the number of particles. The various traces on the right-hand side of eq. (19) must therefore be computed in the form of an expansion in decreasing powers of $N$. Thus, for instance, in order to calculate the term in $\beta$, we use :

$$
\operatorname{Tr}^{\prime} \mathcal{H}_{\mathrm{D}}^{\prime} / \operatorname{Tr}^{\prime} 1=N a_{1}
$$

whereas for calculating the term in $\beta^{2}$ we must compute :

$$
\begin{aligned}
& \operatorname{Tr}^{\prime} \mathcal{H}_{\mathrm{D}}^{\prime} / \operatorname{Tr}^{\prime} 1=N a_{1}+b_{1} \\
& \operatorname{Tr}^{\prime}\left(\mathcal{H}_{\mathrm{D}}^{\prime 2}\right) / \operatorname{Tr}^{\prime} 1=N^{2} a_{2}+N b_{2}, \text { etc. }
\end{aligned}
$$

An explicit calculation shows that $a_{2}=a_{1}^{2}$, as it should.

We show, using a very simple example, how the restricted trace of a product of spin operators can be calculated. We consider the trace of $I_{z}^{i} I_{z}^{j}$, restricted to the subspace $p_{\mathrm{A}}$. We choose as a basis for this subspace a set of kets which correspond to welldefined values of $I_{z}^{l}$ for each spin $I: x$ spins in the state $+\frac{1}{2}$ and $(N-x)$ spins in the state $-\frac{1}{2}$ with :

$$
x=\frac{1}{2} N\left(1+p_{\mathrm{A}}\right) .
$$

There are $C_{N}^{x}=N ! /[x !(N-x) !]$ such kets. The eigenvalues of $I_{z}^{i} I_{z}^{j}$ and their degeneracy are shown below. For instance the number of kets for which $I_{z}^{i}=I_{z}^{j}=+\frac{1}{2}$ is the number for which $(x-2)$ of the $(N-2)$ remaining spins are in the state $+\frac{1}{2}$, that is $C_{N-2}^{x-2}$.

$\begin{array}{cccc}I_{z}^{i} & I_{z}^{j} & I_{z}^{i} I_{z}^{j} & \text { Degeneracy } \\ -\frac{1}{2} & - & - & - \\ +\frac{1}{2} & -\frac{1}{2} & +\frac{1}{4} & C_{N-2}^{x-2} \\ -\frac{1}{2} & +\frac{1}{2} & -\frac{1}{4} & C_{N-2}^{x-1} \\ -\frac{1}{2} & -\frac{1}{2} & -\frac{1}{4} & C_{N-2}^{x-1} \\ & +\frac{1}{4} & C_{N-2}^{x}\end{array}$

Thus, we obtain :

$$
\begin{aligned}
\frac{\operatorname{Tr}^{\prime}\left\{I_{z}^{i} I_{z}^{j}\right\}}{\operatorname{Tr}^{\prime} 1} & =\frac{1}{4}\left\{C_{N-2}^{x}+C_{N-2}^{x-2}-2 C_{N-2}^{x-1}\right\} / C_{N}^{x} \\
& =\frac{1}{4}\left\{(2 x-N)^{2}-N\right\} /[N(N-1)] \\
& =\frac{1}{4}\left\{N^{2} p_{\mathrm{A}}^{2}-N\right\} /[N(N-1)] \\
& =\frac{1}{4} p_{\mathrm{A}}^{2}-\frac{1}{4 N}\left(1-p_{\mathrm{A}}^{2}\right)+\ldots
\end{aligned}
$$

Two alternative methods of calculation are useful. The first one eliminates the temperature and uses instead the entropy as a parameter. This procedure is particularly useful for following the properties of the system during an adiabatic demagnetization, which takes place at constant entropy. The description of this method will however be very brief, since we make little use of it in the following. The second method bypasses the actual computation of the restricted traces through the intermediate use of Lagrange multipliers. For terms of high order in $\beta$ it is considerably simpler than the straightforward computation of eq. (19).

3.1 Elimination OF THE TEMPERATURE. - According to eq. (15) and (16) the energy and the entropy are of the form :

$$
\begin{aligned}
& E=Z\left(p_{\mathrm{A}}, p_{\mathrm{B}}\right)+E_{\mathrm{D}}\left(\beta, p_{\mathrm{A}}, p_{\mathrm{B}}\right) \\
& S=S_{\mathrm{w}}\left(p_{\mathrm{A}}, p_{\mathrm{B}}\right)+s\left(\beta, p_{\mathrm{A}}, p_{\mathrm{B}}\right)
\end{aligned}
$$

where $p_{\mathrm{A}}$ and $p_{\mathrm{B}}$ are well-defined functions of $\beta$, determined by eq. (11). We will use these equations for all values of $p_{\mathrm{A}}$ and $p_{\mathrm{B}}$, that is we define formal functions $E\left(\beta, p_{\mathrm{A}}, p_{\mathrm{B}}\right)$ and $S\left(\beta, p_{\mathrm{A}}, p_{\mathrm{B}}\right)$ which coincide with the energy and the entropy, respectively, in the special case when $p_{\mathrm{A}}$ and $p_{\mathrm{B}}$ satisfy eq. (11).

The expansion in powers of $\beta$ of eq. (15) yields :

$$
E_{\mathrm{D}}=N \sum_{0}^{\infty} \beta^{n} K_{n}\left(p_{\mathrm{A}}, p_{\mathrm{B}}\right)
$$

whence, according to eq. (17) and (18) :

$$
s=N \sum_{1}^{\infty} \frac{n}{n+1} \beta^{n+1} K_{n} .
$$

We can invert eq. (21) and express $\beta$ in the form of a power expansion in $s^{1 / 2}=\left(S-S_{\mathrm{w}}\right)^{1 / 2}$. When inserted into eq. (20) this yields $E_{\mathrm{D}}$ as a function of $S, p_{\mathrm{A}}$ and $p_{\mathrm{B}}$. It can be shown that, as a consequence of eq. (12), (16) and (17), the conditions (10) which determine the values of $p_{\mathrm{A}}$ and $p_{\mathrm{B}}$ are equivalent to :

$$
\left(\frac{\partial E}{\partial p_{\mathrm{A}}}\right)_{S, p_{\mathrm{B}}}=\left(\frac{\partial E}{\partial p_{\mathrm{B}}}\right)_{S, p_{\mathrm{A}}}=0 .
$$

We consider the simplest case, when the expansion (20) is limited to two terms :

$$
E_{\mathrm{D}} / N=K_{0}+\beta K_{1} .
$$

This yields :

$$
s / N=\frac{1}{2} \beta^{2} K_{1} .
$$

If we suppose that $\beta<0$, we obtain :

and

$$
\beta=-\left[\frac{2\left(S-S_{\mathrm{w}}\right)}{N\left|K_{1}\right|}\right]^{1 / 2}
$$

$$
\frac{E}{N}=\frac{Z}{N}+K_{0}-K_{1}\left[\frac{2\left(S-S_{\mathrm{w}}\right)}{N\left|K_{1}\right|}\right]^{1 / 2} .
$$

The practical application of this formalism will not be developed in the present article. 
It can be noted that, one can also express the entropy as a function of energy. In the approximation used above, we obtain :

$$
\beta=\left(\frac{E_{\mathrm{D}}}{N}-K_{0}\right) / K_{1}
$$

and :

$$
S=S_{\mathrm{w}}+\frac{1}{2} N\left(\frac{E_{\mathrm{D}}}{N}-K_{0}\right)^{2} / K_{1} .
$$

It can be shown that eq. (10) are equivalent to :

$$
\left(\frac{\partial S}{\partial p_{\mathrm{A}}}\right)_{E, p_{\mathrm{B}}}=\left(\frac{\partial S}{\partial p_{\mathrm{B}}}\right)_{E, p_{\mathrm{A}}}=0,
$$

which yields the values of $p_{\mathrm{A}}$ and $p_{\mathrm{B}}$.

This calculation, when applied to the case of ferromagnetism, is identical with that derived by Heisenberg using a different approximation [10, 11]. Starting from a completely different point of view, Heisenberg assumes that the density of states, whose logarithm is proportional to the entropy, is within a subspace of given polarization, a Gaussian function of the correlation energy, which he fits to the first moment $\left(N K_{0}\right)$ and to the second moment $\left(-N K_{1}\right)$ of the energy spectrum within that subspace.

The Kirkwood approximation then appears as an extension of the Heisenberg approximation : Firstly, it allows the inclusion of higher order terms in correlation energy, and secondly it can be used for antiferromagnetism; that is, when the spin-spin Hamiltonian has non-vanishing matrix elements between different subspaces.

3.2 Use OF LaGRANGe MULTIPLIERS. - Instead of performing the trace calculations within a subspace with well-defined values of $p_{\mathrm{A}}$ and $p_{\mathrm{B}}$, we can use a weighted distribution of subspaces. That is, we replace the calculation of :

$$
\operatorname{Tr}^{\prime}\left\{\exp \left(-\beta \mathcal{H}_{\mathrm{D}}^{\prime}\right)\right\} / \operatorname{Tr}^{\prime} 1
$$

by that of :

$$
\begin{aligned}
\operatorname{Tr}\left\{\exp \left(\lambda I_{z}+\mu S_{z}\right) \exp \left(-\beta \mathcal{H}_{\mathrm{D}}^{\prime}\right)\right\} / \operatorname{Tr} \times \\
\times\left\{\exp \left(\lambda I_{z}+\mu S_{z}\right)\right\} .
\end{aligned}
$$

This is completely analogous to the passage from the microcanonical distribution to the canonical distribution in thermodynamics. In practice, it proves simpler to calculate the logarithmic derivative of the expression (27) with respect to $\beta$ than the expression (27) itself. This derivative is to within a change of sign, equal to the dipolar energy $E_{\mathrm{D}}$ corresponding to the density matrix :

$$
\sigma=\exp \left(\lambda I_{z}+\mu S_{z}\right) \exp \left(-\beta \mathcal{H}_{\mathrm{D}}^{\prime}\right) / \operatorname{Tr}\{\cdots\} .
$$

This calculation can be carried out using a diagrammatic method very similar to that of Stinchcombe et al. [12-14], which yields $E_{\mathrm{D}}$ in the form of a power expansion with respect to $\beta$. The Lagrange multipliers $\lambda$ and $\mu$ are determined by the conditions :

and :

$$
\begin{aligned}
& \operatorname{Tr}\left\{\sigma I_{z}\right\}=\frac{1}{2} N p_{\mathrm{A}} \\
& \operatorname{Tr}\left\{\sigma S_{z}\right\}=\frac{1}{2} N p_{\mathbf{B}} .
\end{aligned}
$$

By expressing, through eq. (29), $\lambda$ and $\mu$ as a function of $\beta, p_{\mathrm{A}}$ and $p_{\mathrm{B}}$ and inserting the results in the expression for $E_{\mathrm{D}}(\beta, \lambda, \mu)$, we obtain for the dipolar energy a form identical with eq. (20). The calculations are then pursued as before.

This method is not significantly better than the restricted-trace calculation for computing the coefficients $K_{0}$ and $K_{1}$. It is however, already very much simpler for calculating $K_{2}$.

\section{Physical properties of a nuclear antiferromagnet.} - We report in this section the predictions drawn from the Kirkwood approximation for various properties of the antiferromagnetic structure shown in figure 1. The calculations are made for a spherical sample, and the figures correspond to the fluorine spin system in $\mathrm{CaF}_{2}$.
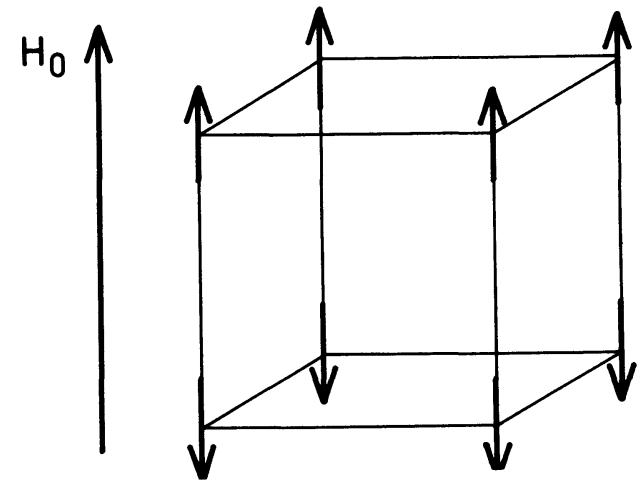

FIG. 1. - Antiferromagnetic structure of $\mathrm{CaF}_{2}$ after adiabatic demagnetization in the rotating frame, with $H_{0} / /[100]$ and $T<0$.

The truncated dipole-dipole Hamiltonian is written as follows

$$
\begin{aligned}
\mathcal{H}_{\mathrm{D}}^{\prime}=\frac{1}{2} \sum_{i j} & B_{i j}\left[2 I_{z}^{i} I_{z}^{j}-\frac{1}{2}\left(I_{+}^{i} I_{-}^{j}+I_{-}^{i} I_{+}^{j}\right)\right]+ \\
& +\frac{1}{2} \sum_{\mu \nu} B_{\mu v}\left[2 S_{z}^{\mu} S_{z}^{v}-\frac{1}{2}\left(S_{+}^{\mu} S_{-}^{v}+S_{-}^{\mu} S_{+}^{v}\right)\right] \\
& +\sum_{i \mu} C_{i \mu}\left[2 I_{z}^{i} S_{z}^{\mu}-\frac{1}{2}\left(I_{+}^{i} S_{-}^{\mu}+I_{-}^{i} S_{+}^{\mu}\right)\right]
\end{aligned}
$$

where the spins $I^{i}$ and $S^{\mu}$ belong to the sublattices $A$ and $\mathcal{B}$, respectively, and the coefficients have the well-known form :

and

$$
\begin{aligned}
& B_{i j}=\frac{1}{2} \gamma^{2} \hbar\left(1-3 \cos ^{2} \theta_{i j}\right) r_{i j}^{-3}, \\
& B_{\mu \nu}=\frac{1}{2} \gamma^{2} \hbar\left(1-.3 \cos ^{2} \theta_{\mu \nu}\right) r_{\mu \nu}^{-3}, \\
& C_{i \mu}=\frac{1}{2} \gamma^{2} \hbar\left(1-3 \cos ^{2} \theta_{i \mu}\right) r_{i \mu}^{-3} .
\end{aligned}
$$


We have calculated the dipolar energy up to the term in $\beta^{2}$ :

$$
E_{\mathrm{D}} / N=K_{0}+\beta K_{1}+\beta^{2} \dot{K}_{2}+\cdots .
$$

The calculations are complicated and will not be reproduced here. The form of the coefficients as well as their computed values for a simple cubic lattice of parameter $a$, of spin $\frac{1}{2}$ of gyromagnetic ratio $\gamma$, are the following.

i)

$$
K_{0}=q\left(p_{\mathrm{A}}-p_{\mathrm{B}}\right)^{2} / 8
$$

where the Weiss field coefficient $q$ is equal to :

$$
\begin{aligned}
q & =\sum_{i} B_{i j}-C_{i \mu} \\
& =9.687 \gamma^{2} \hbar /\left(2 a^{3}\right)
\end{aligned}
$$

ii) $K_{1}=-\frac{1}{16}\left\{\mathfrak{D}\left[6+\left(p_{\mathrm{A}}^{2}+p_{\mathrm{B}}^{2}\right)^{2}-4\left(p_{\mathrm{A}}^{2}+p_{\mathrm{B}}^{2}\right)\right.\right.$

$$
\begin{aligned}
& \left.-\frac{1}{2}\left(p_{\mathrm{A}}+p_{\mathrm{B}}\right)^{2}\right]+ \\
& \left.+\varepsilon\left[\left(p_{\mathrm{A}}^{2}-p_{\mathrm{B}}^{2}\right)^{2}-\frac{1}{2}\left(p_{\mathrm{A}}-p_{\mathrm{B}}\right)^{2}\right]\right\}
\end{aligned}
$$

with :

$$
\begin{aligned}
\mathcal{D} & =\sum_{i}\left(B_{i j}^{2}+C_{i \mu}^{2}\right) \\
& =13.357\left[\gamma^{2} \hbar /\left(2 a^{3}\right)\right]^{2},
\end{aligned}
$$

and

$$
\begin{aligned}
\mathcal{E} & =\sum_{i}\left(B_{i j}^{2}-C_{i \mu}^{2}\right) \\
& =-3.396\left[\gamma^{2} \hbar /\left(2 a^{3}\right)\right]^{2}
\end{aligned}
$$

iii) $K_{2}=\frac{1}{64} \times$

$\times\left\{X_{1}\left[\left(1-p_{\mathrm{A}}^{2}\right)^{2}\left(8 p_{\mathrm{A}}^{2}-3\right)+\left(1-p_{\mathrm{B}}^{2}\right)^{2}\left(8 p_{\mathrm{B}}^{2}-3\right)\right]\right.$

$+X_{2}\left[2\left(1-p_{\mathrm{A}}^{2}\right)\left(1-p_{\mathrm{B}}^{2}\right)\left(8 p_{\mathrm{A}} p_{\mathrm{B}}-1\right)-4\left(1-p_{\mathrm{A}} p_{\mathrm{B}}\right)^{2}\right]$

$+X_{3}\left[4\left[\left(1-p_{\mathrm{A}}^{2}\right)^{3}+\left(1-p_{\mathrm{B}}^{2}\right)^{3}\right]+p_{\mathrm{A}}^{2}+p_{\mathrm{B}}^{2}-2\right]$

$+X_{4}\left[\left(p_{\mathrm{A}}^{2}+p_{\mathrm{B}}^{2}-2\right)\left[1-12\left(1-p_{\mathrm{A}}^{2}\right)\left(1-p_{\mathrm{B}}^{2}\right)\right]\right.$

$\left.\left.-4\left(1-p_{\mathrm{A}} p_{\mathrm{B}}\right)\right]\right\}$

with :

$$
\begin{aligned}
X_{1} & =\sum_{i} B_{i j}^{3}=4.141\left[\gamma^{2} \hbar /\left(2 a^{3}\right)\right]^{3}, \\
X_{2} & =\sum_{i} C_{i \mu}^{3}=-16.046\left[\gamma^{2} \hbar /\left(2 a^{3}\right)\right]^{3}, \\
X_{3} & =\sum_{j l} B_{i j} B_{j l} B_{l i} \\
& =18.393\left[\gamma^{2} \hbar /\left(2 a^{3}\right)\right]^{3}, \\
X_{4} & =\sum_{j \mu} B_{i j} C_{j \mu} C_{\mu i} \\
& =1.902\left[\gamma^{2} \hbar /\left(2 a^{3}\right)\right]^{3} .
\end{aligned}
$$

Eq. (11) become :

$$
\begin{aligned}
\tanh ^{-1}\left(p_{\mathrm{A}}\right)=-\frac{1}{2} \beta \Delta-\frac{1}{4} \beta q\left(p_{\mathrm{A}}-p_{\mathrm{B}}\right)- & \\
& -\frac{\beta^{2}}{2} \frac{\partial K_{1}}{\partial p_{\mathrm{A}}}-\frac{\beta^{3}}{3} \frac{\partial K_{2}}{\partial p_{\mathrm{A}}}
\end{aligned}
$$

$$
\begin{aligned}
\tanh ^{-1}\left(p_{\mathrm{B}}\right)=-\frac{1}{2} \beta \Delta+\frac{1}{4} \beta q( & \left.p_{\mathrm{A}}-p_{\mathrm{B}}\right)- \\
& -\frac{\beta^{2}}{2} \frac{\partial K_{1}}{\partial p_{\mathrm{B}}}-\frac{\beta^{3}}{3} \frac{\partial K_{2}}{\partial p_{\mathrm{B}}} .
\end{aligned}
$$

We have used as a rule two successive approximations. The first-order approximation neglects the terms in $K_{2}$, and the second-order approximation includes them. In the following we give the detailed formulae only for the first-order approximation, which is the simplest one.

According to eq. (34) the derivatives of $K_{1}$ are

$$
\begin{array}{r}
\frac{\partial K_{1}}{\partial p_{\mathrm{A}}}=-\frac{1}{16}\left\{\mathfrak{D}\left[4 p_{\mathrm{A}}\left(p_{\mathrm{A}}^{2}+p_{\mathrm{B}}^{2}\right)-9 p_{\mathrm{A}}-p_{\mathrm{B}}\right]+\right. \\
\left.+\varepsilon\left[4 p_{\mathrm{A}}\left(p_{\mathrm{A}}^{2}-p_{\mathrm{B}}^{2}\right)-p_{\mathrm{A}}+p_{\mathrm{B}}\right]\right\}
\end{array}
$$

$$
\begin{aligned}
\frac{\partial K_{1}}{\partial p_{\mathrm{B}}}=-\frac{1}{16}\{\mathfrak{D}[4 & \left.p_{\mathrm{B}}\left(p_{\mathrm{A}}^{2}+p_{\mathrm{B}}^{2}\right)-9 p_{\mathrm{B}}-p_{\mathrm{A}}\right]+ \\
& \left.+\delta\left[4 p_{\mathrm{B}}\left(p_{\mathrm{B}}^{2}-p_{\mathrm{A}}^{2}\right)+p_{\mathrm{A}}-p_{\mathrm{B}}\right]\right\} .
\end{aligned}
$$

The various properties investigated are listed below.

4.1 ENERGY, ENTROPY AND SUBLATTICE POLARIZATIONS IN ZERO FIELD. - We will first discuss the sublattice polarizations. This is the most fundamental property of antiferromagnets. Although they have not yet been experimentally measured, their study is necessary for checking the consistency of the approximation as well as for deriving the critical values of temperature, entropy and energy.

When $\Delta=0$ it is found that the solution of eq. (36) which maximizes $\ln Z$ does indeed correspond to $p_{\mathrm{A}}=-p_{\mathrm{B}}=p$. We obtain, for the first order approximation :

$\tanh ^{-1}(p)=-\frac{1}{2} \beta q p-\frac{1}{16} \beta^{2} p\left[4 \mathfrak{D}\left(1-p^{2}\right)+\varepsilon\right]$.

This yields a non-vanishing value of $p$ only if :

$$
\begin{aligned}
\tanh ^{-1}(p) / p=- & \frac{1}{2} \beta q-\frac{1}{16} \times \\
& \times \beta^{2}\left[4 D\left(1-p^{2}\right)+\varepsilon\right] \geqslant 1 .
\end{aligned}
$$

The critical inverse temperature $\beta_{\mathrm{c}}$ corresponds to :

$$
-\frac{1}{2} \beta_{\mathrm{c}} q-\frac{1}{16} \beta_{\mathrm{c}}^{2}[4 \mathrm{D}+\varepsilon]=1 \text {. }
$$


whereas the Weiss-field approximation corresponds to :

$$
-\frac{1}{2} \beta_{\mathrm{c}} q=1 \text {. }
$$

For $\beta$ slightly below $\beta_{\mathrm{c}}$ (we recall that in the present case we have $\beta<0), p$ is small and we have :

$$
\tanh ^{-1}(p) \simeq p+\frac{1}{3} p^{3}
$$

whence, according to eq. (38)

$$
p \propto\left(\beta_{\mathrm{c}}-\beta\right)^{1 / 2} .
$$

This exhibits the qualitatively correct feature that :

$$
\mathrm{d} p / \mathrm{d} \beta=\infty \quad \text { at } \beta=\beta_{\mathrm{c}} .
$$

However, the correctness of the critical exponent $\frac{1}{2}$ is much more dubious. In systems of spins coupled by short-range interactions it is known to be wrong.

The energy and the entropy per spin are, according to eq. (23), (24), (32) and (34), equal to :

$E / 2 N=\frac{1}{4} q p^{2}-\frac{1}{16} \beta\left\{\mathfrak{D}\left[3-4 p^{2}+2 p^{4}\right]-\varepsilon p^{2}\right\}$

$$
\begin{array}{rl}
S / 2 N=S_{\mathrm{w}} / 2 & N-\frac{1}{32} \beta^{2} \times \\
& \times\left\{\mathfrak{D}\left[3-4 p^{2}+2 p^{4}\right]-\varepsilon p^{2}\right\} .
\end{array}
$$

We list in table I the critical values of the temperature, of the entropy, and of the initial polarization prior to the adiabatic demagnetization in the rotating frame. As discussed in reference [5], this initial polarization is simply related to the entropy of the system. The critical values are given for the 1 st and 2 nd order restricted-trace approximations and also, for comparison, for the Weiss-field and the Random-Phase approximations. Also included are the numerical values of $T_{\mathrm{c}}$ and $E_{\mathrm{c}}$ for calcium fluoride.

Eq. (38), (41) and (42) exhibit the inadequacy of the present method close to zero temperature (i.e. for large values of $|\beta|)$. This is indeed to be expected from an approximation method which uses a power expansion in $\beta$ for the correlation energy and entropy. Aćcording to eq. (38), $p \rightarrow 1$ when $|\beta| \rightarrow \infty$, whereas, when $|\beta|$ is large, that is $p \simeq 1$, eq. (41) and (42) yield :

$$
\begin{aligned}
& E / 2 N \simeq \frac{1}{4} q-\frac{1}{16} \beta[\mathfrak{D}-\varepsilon] \\
& S / 2 N \simeq S_{\mathrm{w}} / 2 N-\frac{1}{32} \beta^{2}[\mathfrak{D}-\varepsilon] .
\end{aligned}
$$

The energy increases with $\beta$ beyond limits whereas the entropy becomes negative, which is unacceptable. The same qualitative features occur for the 2 nd order approximation. The restricted-trace approximation must therefore be limited to values of $|\beta|$ much lower than the value which makes the entropy to vanish.

Figure 2 reproduces the variation of the sublattice polarizations according to the four approximations cited in table I. The variations predicted by the restricted-trace approximations are qualitatively as expected for an antiferromagnet. The sublattice polarizations, extrapolated to the initial polarization $p_{\mathrm{i}}=1$ cannot be taken too seriously, as noted above. Their values, $96 \%$ (1st order) and $94 \%$ (2nd order) are slightly smaller than the value $p_{\mathrm{A}}=98.5 \%$

\begin{tabular}{|c|c|c|c|c|c|}
\hline \multirow[b]{2}{*}{ Approximation } & \multicolumn{2}{|c|}{$T_{\mathrm{c}}$} & \multicolumn{2}{|c|}{$E_{\mathrm{c}}$} & $p_{\text {ic }}$ \\
\hline & $\frac{\gamma^{2} \hbar}{2 k_{\mathrm{B}} a^{3}}$ & $\mu K\left(\mathrm{CaF}_{2}\right)$ & $\frac{\gamma^{2} \hbar}{2 a^{3}}$ & $k H_{z}\left(\mathrm{CaF}_{2}\right)$ & $\%$ \\
\hline - & & & & & - \\
\hline Weiss & -4.844 & -0.614 & 0 & 0 & 0 \\
\hline RPA & -4.654 & -0.590 & 0.18 & 0.475 & 11 \\
\hline R.T. 1st Ord. & -4.078 & -0.517 & 0.615 & 1.62 & 38 \\
\hline R.T. 2nd Ord. & -4.034 & -0.511 & 0.725 & 1.91 & 43 \\
\hline
\end{tabular}
predicted by RPA and spin-wave approximations, which is expected to be correct.

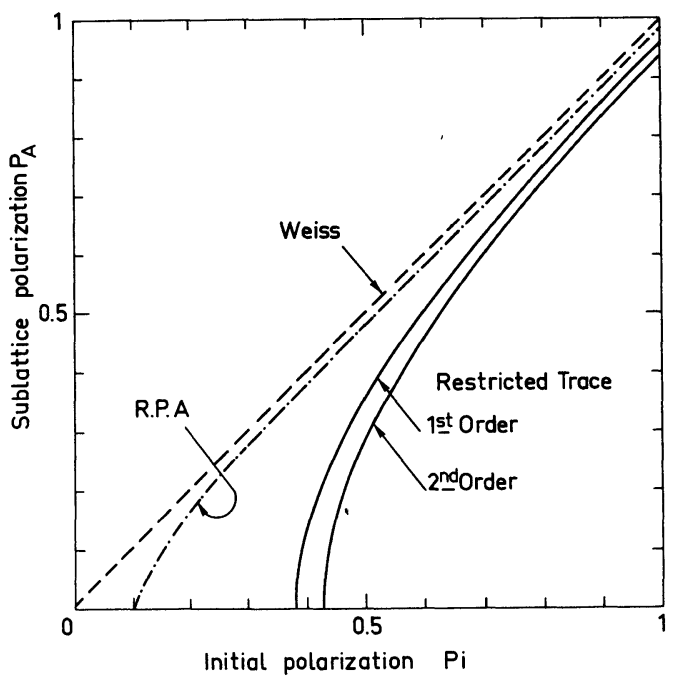

FIG. 2. - Sublattice polarizations in zero field as a function of initial polarization according to Weiss-field, R.P.A., and 1st and 2nd order Restricted-trace approximations.

TABLE I 
4.2 TRANSVERSE SUSCEPTIBILITY IN ZERO FIELD. When an $\mathrm{rf}$ field is applied at a distance $\Delta$ from resonance, it appears static in the rotating frame. The corresponding effective Hamiltonian is :

$$
\mathscr{H}=\Delta\left(I_{z}+S_{z}\right)+\omega_{1}\left(I_{x}+S_{x}\right)+H_{\mathrm{D}}^{\prime} .
$$

If $\omega_{1}$ is small, the net magnetization along the rf field is proportional to $\omega_{1}$. We define the transverse susceptibility_by :

$$
\chi_{\perp}=\frac{1}{2 \omega_{1}}\left(p_{\mathrm{Ax}}+p_{\mathrm{B} x}\right)
$$

with

$$
p_{\mathrm{A} x}=2\left\langle I_{x}^{i}\right\rangle \text { and } p_{\mathrm{B} x}=2\left\langle S_{x}^{\mu}\right\rangle .
$$

The calculations are complicated by the fact that the subspaces one must use for computing the restricted traces correspond to polarizations of the spins $I$ and $S$ which are not aligned with the direction $\mathrm{O} z$ but are tilted away by angles $\theta_{\mathrm{A}}$ and $\theta_{\mathrm{B}}$ a priori different. One must then maximize $\ln Z$ with respect to four parameters : $p_{\mathrm{A}}, p_{\mathrm{B}}, \theta_{\mathrm{A}}$ and $\theta_{\mathrm{B}}$. The situation is somewhat simpler in zero effective field : the magnitudes of the two sublattice polarizations are then equal, and the tilting angles $\theta_{\mathrm{A}}$ and $\theta_{\mathrm{B}}$ are also equal, so that only two parameters remain. The calculations are however still much more complicated than in the absence of a rf field. They were only performed to the 1 st order approximation, and will not be reproduced here. The calculations are made separately for the paramagnetic and the antiferromagnetic states.

In the paramagnetic state, the only non-vanishing polarization component is along $\omega_{1}$. It is equal for all spins. The reduced-trace approximation in that case yields :

$$
\chi_{\perp}(\text { para })=-\frac{1}{2} \beta /\left[1-\frac{1}{16} \beta^{2} D\right] .
$$

This approximation is very poor in that case since, when expanded in powers of $\beta$ and compared with an exact high-temperature expansion [16], the term in $\beta^{3}$ has the wrong sign.

In the antiferromagnetic state, the tilting angle $\theta$ is always very small, except in the immediate vicinity of the transition. We can then use a power expansion with respect to $\theta$. The final result is :

$$
\begin{aligned}
\chi_{\perp}(\mathrm{AF})= & \left(1+\frac{\beta q}{2}\right) /\left\{q\left(1+\frac{\beta q}{2}\right)+\frac{1}{8} \beta \times\right. \\
& \left.\times\left[\mathfrak{D}\left(5-4 p^{2}\right)+\varepsilon\left(1-2 p^{2}\right)\right]\right\} .
\end{aligned}
$$

Eq. (45) extrapolates to the same value as eq. (44) when $\beta$ tends toward $\beta_{\mathrm{c}}$.

Figures 3 and 4 show the variations of $\chi_{\perp}$ given by eq. (44) and (45) as a function of initial polarization and dipolar energy, respectively, together with the

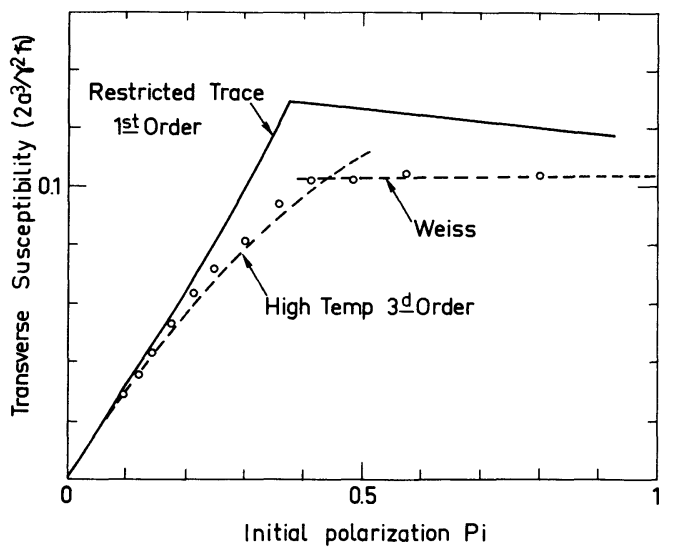

FIG. 3. - Transverse susceptibility as a function of initial polarization according to Weiss-field, High-temperature, and 1st order Restricted-trace approximations, together with experimental results in $\mathrm{CaF}_{2}$.

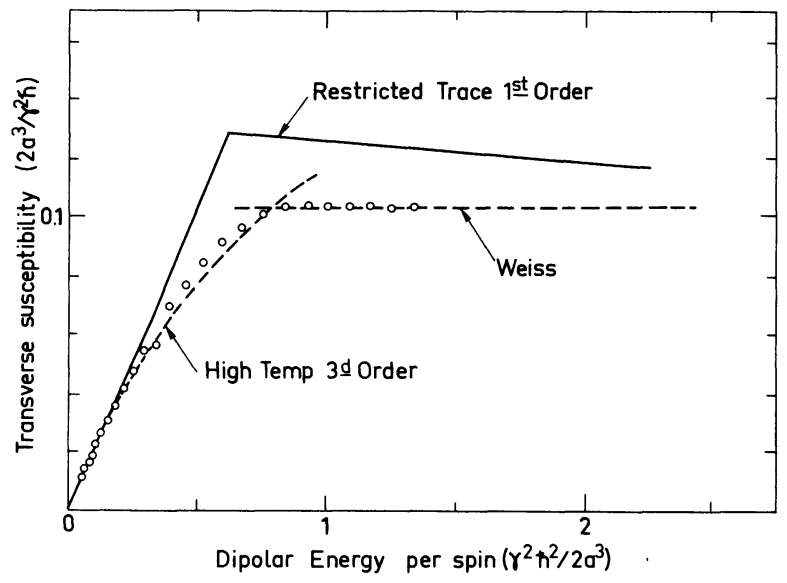

FIG. 4. - Transverse susceptibility as a function of dipolar energy, according to the same approximations as in figure 3 , together with experimental results in $\mathrm{CaF}_{2}$.

experimental results and the predictions of Weissfield and high-temperature approximations. In the paramagnetic region, the present theory gives an erroneous account of the non-linear variation of $\chi_{\perp}$; in the antiferromagnetic region $\chi_{\perp}$, which varies slightly, is about $25 \%$ higher than predicted by the Weiss-field approximation.

The latter value is expected to be closer to reality, on the following grounds. Firstly, the experimental values of $\chi_{\perp}$ are nearly constant in the antiferromagnetic phase, in accordance with the Weiss-field, RPA and Spin-wave theories; secondly the Weissfield value of $\chi_{\perp}$ coincides with that predicted by the RPA and Spin-wave approximations, which are expected to be accurate at zero temperature.

When the experimental values of $\chi_{\perp}$ in the plateau are adjusted to the Weiss-field prediction, the results exhibit a better agreement with the approximations of reference [5] than with the present one. There is however a qualitative agreement of the predictions 
with the results, which shows in particular that the critical values of $E_{\mathrm{c}}$ and $p_{\text {ic }}$ reported in table I are of the right order of magnitude.

4. 3 LONGITUDINAL SUSCEPTIBILITY IN ZERO FIELD. - When the longitudinal effective field is small, the longitudinal magnetization is proportional to it. We define the longitudinal susceptibility $\chi_{\|}$through :

$$
\chi_{\|}=\frac{1}{2 \Delta}\left(p_{\mathrm{A}}+p_{\mathrm{B}}\right)
$$

It is calculated as follows. We write

$$
p_{\mathrm{A}}=p+\varepsilon \text { and } p_{\mathrm{B}}=-p+\varepsilon^{\prime}
$$

we insert these values into eq. (36), which we expand to the first order in $\varepsilon, \varepsilon^{\prime}$ and $\Delta$. We find that $\varepsilon=\varepsilon^{\prime}$. The first-order restricted-trace approximation yields :

$$
\begin{array}{r}
\chi_{\|}=\frac{\varepsilon}{\Delta}=-\frac{1}{2} \beta\left(1-p^{2}\right) /\left\{1-\frac{1}{16} \beta^{2}\left(1-p^{2}\right) \times\right. \\
\left.\times\left|\mathfrak{D}\left(4 p^{2}-5\right)+8 \varepsilon p^{2}\right|\right\} .
\end{array}
$$

The variation of $\chi_{\|}$as a function of energy is displayed on figure 5 , both for the 1st order and the 2nd order approximations, together with the experimental results and the predictions of high-temperature, Weiss-field and random phase approximations. The experimental values have been calibrated against those of $\chi_{\perp}$ (Fig. 4) [4]. Here again, the present approximations are in qualitative agreement with the experimental results but, if we assume that the calibration of $\chi_{\|}$is correct, they are not so good as the combination of high-temperature and Weiss-field or RPA approximations. Furthermore, the 2nd order approximation yields little improvement, if any, over the 1st order one.

The calculations have been limited to the value of $\beta$ for which the entropy vanishes. In this limit the longitudinal susceptibility $\chi_{\|}$is not zero, contrary to a

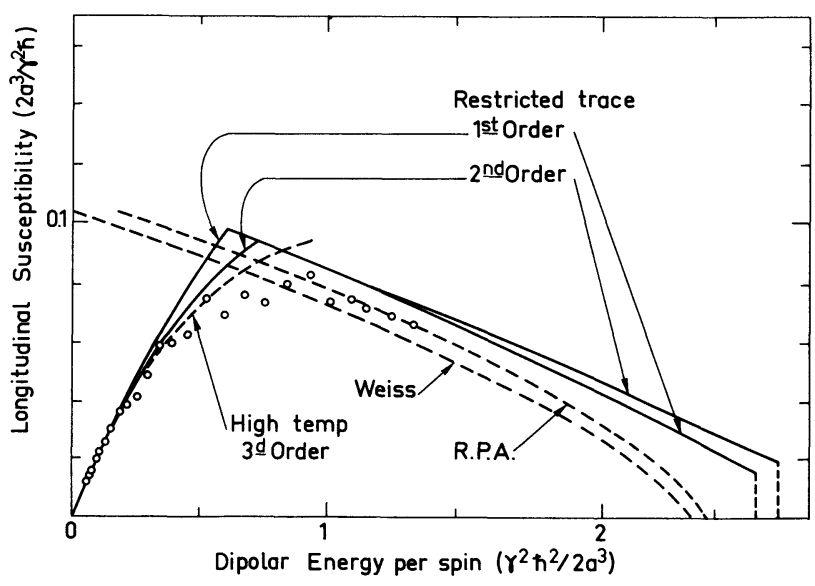

FIG. 5. - Longitudinal susceptibility as a function of dipolar energy, according to High-temperature, Weiss-field, R.P.A., and 1st and 2nd order Restricted-trace approximations, together with experimental results in $\mathrm{CaF}_{2}$. well-known property of antiferromagnets which is correctly predicted by the Weiss-field, random phase and spin wave theories. This is another manifestation of the inadequacy of the restricted-trace expansion at low temperature.

We will not consider in this article the predictions for the field of transition from paramagnetism to anti-ferromagnetism, whose experimental investigation is not yet completed.

5. Conclusion. - We have used in this work the restricted trace approximation to predict several properties of a nuclear antiferromagnet.

This approximation is successful in accounting in a qualitative way for the experimental results : transverse and longitudinal susceptibilities as a function of entropy or energy, which is outside of the reach of the Weiss-field approximation. The agreement is however not perfect, and the departure of the theoretical predictions from the experimental results is distinctly larger. than the experimental uncertainty. In that respect, the second-order approximation does not yield any definite improvement over the firstorder one, while involving much heavier calculations. Also we have insisted in the text on the drawbacks of this approximation : the inability to account for the nonlinearity of $\chi_{\perp}$ in the paramagnetic state and non-physical behaviour at low temperature.

Furthermore, one might question the usefulness of the restricted-trace approximation in view of the rather surprising result that we obtain a better agreement with experiment from a high temperature expansion in the paramagnetic phase, and from the Weiss-field approximation in the antiferromagnetic phase. One must note however that the high-temperature and the Weiss-field approximations extend to domains of entropy and energy where they are completely erroneous. The agreement with experiment is obtained by going over from one type of behaviour to the other one, which is inconsistent. A striking example of this inconsistency is provided by the variation of $\chi_{\|}$with energy (Fig. 5). At the crossing point between the high-temperature and the Weiss-field approximations the value of $\chi_{\|}$, as computed by the high-temperature expansion corresponds to a paramagnetic state with no long-range order, whereas the same value of $\chi_{\|}$corresponds in the Weiss-field approximation to a sublattice polarization of $52 \%$.

The great advantage of the restricted trace approximation, despite its defects, is that it covers consistently with a single formalism both domains of paramagnetism and ordering. It also takes into account, if only partially, the correlation between spins in the ordered phase which, for the study of some problems is a necessity.

Examples will be given in forthcoming articles.

Since the second-order approximation is not decisively better than the first-order one, a much more elaborate approach would probably be necessary 
to obtain a real improvement. In the absence of such an approach, it is amply justified, and sufficient, to use the first-order restricted trace approximation to obtain, through a very simple calculation, the gross features of the phenomena associated with nuclear dipolar magnetic transitions.
Acknowledgments. - We acknowledge the contribution through many discussions of Professor A. Abragam and Drs M. Chapellier and J. F. Jacquinot.

\section{References}

[1] Chapellier, M., Goldman, M., Vu Hoang Chau and Abragam, A., C. R. Hebd. Séan. Acad. Sci. 268 (1969) 1530.

[2] Chapellier, M., Goldman, M., Vu Hoang Chau and AbraGaM, A., J. Appl. Phys. 41 (1970) 849.

[3] Jacquinot, J. F., Wenckebach, W. T., Chapellier, M., Goldman, M. and Abragam, A., C. R. Hebd. Séan. Acad. Sci. B 278 (1974) 93.

[4] Jacquinot, J. F., Chapellier, M. and Goldman, M., Phys. Lett. 48A (1974) 303.

[5] Goldman, M., Chapellier, M., Vu Hoang Chau and AbraGAM, A., Phys. Rev. B 10 (1974) 226.

[6] Kirkwood, J. G., J. Chem. Phys. 6 (1938) 70.

[7] Brout, R., Phase Transitions (Benjamin, New York) 1965, p. 12 .
[8] Bragg, W. and Williams, E., Proc. R. Soc. A 145 (1934) 609.

[9] BROUT, R., loc. cit. p. 10.

[10] Heisenberg, W., Z. Physik 49 (1928) 619.

[11] VAN Vleck, J. H., The Theory of Electric and Magnetic Susceptibilities (Oxford University Press) 1932, p. 326.

[12] Stinchcombe, R., Horwitz, G., Englert, F. and Brout, R., Phys. Rev. 130 (1963) 155.

[13] Brout, R. in Rado, G. T. and SuHL, H., Magnetism (Academic Press, New York) 1964, Vol. IIA, p. 43.

[14] Brout, R., Phase Transitions, loc. cit., ch. 2 and 5.

[15] Vu Hoang Chau, Thesis, C.N.R.S. No 708 (Orsay 1970).

[16] Goldman, M., Jacquinot, J. F., Chapellier, M. and Vu Hoang Chau, J. Magn. Reson. 18 (1975) 22. 